\title{
The impact on the deep leap movement before and after the exercise fatigue based on sports biomechanics.
}

\section{Lixun $\mathrm{Hu}^{*}$}

Department of Physical Education, Shaanxi University of Chinese Medicine, 712046, PR China

\begin{abstract}
With the increasing attention to the health of modern people, the proportion of sports in life is getting bigger and bigger. However, the effects of exercise induced fatigue on motor movements are less studied. Based on the introduction of sports biomechanics and sports fatigue, the impact on the deep leap movement before and after the exercise fatigue based on sports biomechanics was studied, and the influence on the structure of the deep leap action was taken as an example to validate the scheme created. The results show that the range of study in this paper is consistent with sports biomechanics.
\end{abstract}

Keywords: Sports biomechanics, Sports fatigue; Deep leap action, Influence.

Accepted on April 29, 2017

\section{Introduction}

With the continuous improvement of living standards, people pay more and more attention to their health problems; this makes the application of sports medicine in daily life more and more. Yoshikawa and others believe that exercise fatigue not only is physical fatigue, but also includes psychological fatigue [1]. In this condition, the storage capacity of muscles is decreased, its ability of dispersing energy is also decreased, the muscles are unable to withstand the same load, so as to produce movement deformation, and increase the risk of muscle strain. The athlete's athletic ability is closely related to the exercise fatigue. Through the study of sports fatigue, it is found that the effect of sports fatigue on the deformation is very significant. At the same time, the relevant parameters of the movement will continue to decline, the body's internal metabolism increases, thereby resulting in a certain amount of bone and muscle fatigue. Based on this, this paper discusses the sports biomechanics and sports fatigue, and studies the impact on the deep leap movement before and after the exercise fatigue based on sports biomechanics.

\section{State of the Art}

\section{Sports biomechanics}

Sports Biomechanics is mainly to study the cross analysis based on biology and mechanics of biology and mechanics in the process of movement (Figure 1). Up to now, our country has made some achievements in the equipment of sports biomechanics. However, there are still some problems in the construction of the standard subject. In November 2006, the Eleventh National Conference on Sports Biomechanics was held in Chengdu Sport University, the General Assembly reflected the overall level of China's current sports biomechanics, but also planned its development direction.

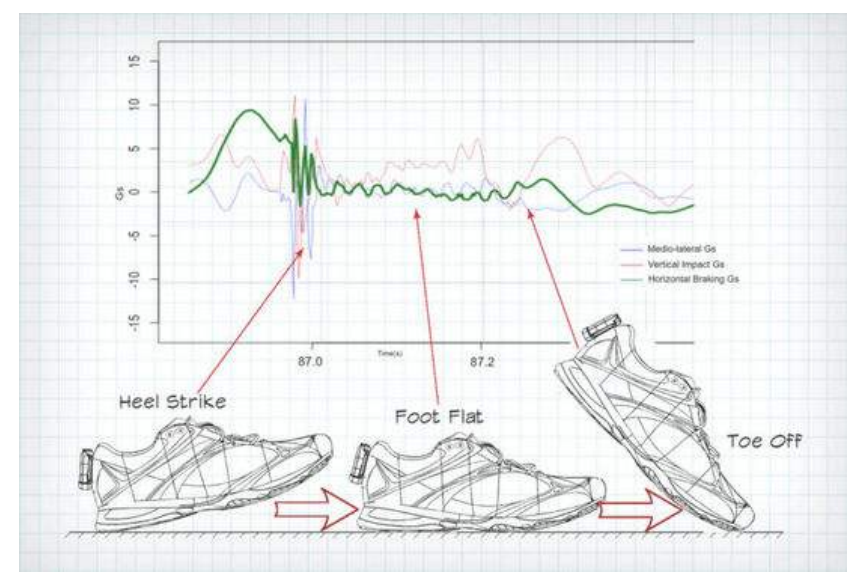

Figure 1. Schematic diagram of sports biomechanics.

\section{Exercise fatigue}

Sports fatigue refers to the physiological phenomenon of the maximum contraction or the maximum output power of a muscle during exercise. In the physiological process of the organism, the movement can't keep the body at a certain level. Exercise reduces the body's ability to work, but the original state can be restored after a period of rest, which is harmless to the body. But excessive physical fatigue can change the body's parameters; the original fatigue has not yet returned to the new fatigue; this is likely to cause damage during exercise, while reducing its ability to recover. Later research shows that sports fatigue not only can cause physical fatigue, but also psychological fatigue. The body fatigue will reduce the speed of action, the sensitivity and the coordination ability [2]. Mental fatigue is a psychological state of fatigue during 
exercise; this is mainly reflected in the movement of the body in the operation of the brain, which will affect the working state of the brain, and further lead to physical fatigue.

\section{Deep leap motion}

Deep leap action refers to jump from a height and then jump to another height. In this process, both legs should be flexible, and must do a "ground" action, this action does not need to load, but the feet should be able to buffer ground, so the ground should not be too hard. This action can improve the muscle strength of the legs of the athletes, and improve leg explosive power and the overall performance. The essence of the deep leap is the mechanical stimulation of the muscles, transient mechanical shock can enhance the ability of the muscles to withstand tensile load, and improve the ability of the nervous system. However, there is a difference between deep jumping exercises before and after exercise induced fatigue.

\section{Methodology}

Sports fatigue can reduce the output power of muscle or body. The program shown below shows the effects on the structure of the deep leap movement before and after the exercise fatigue based on sports biomechanics (Figure 2).

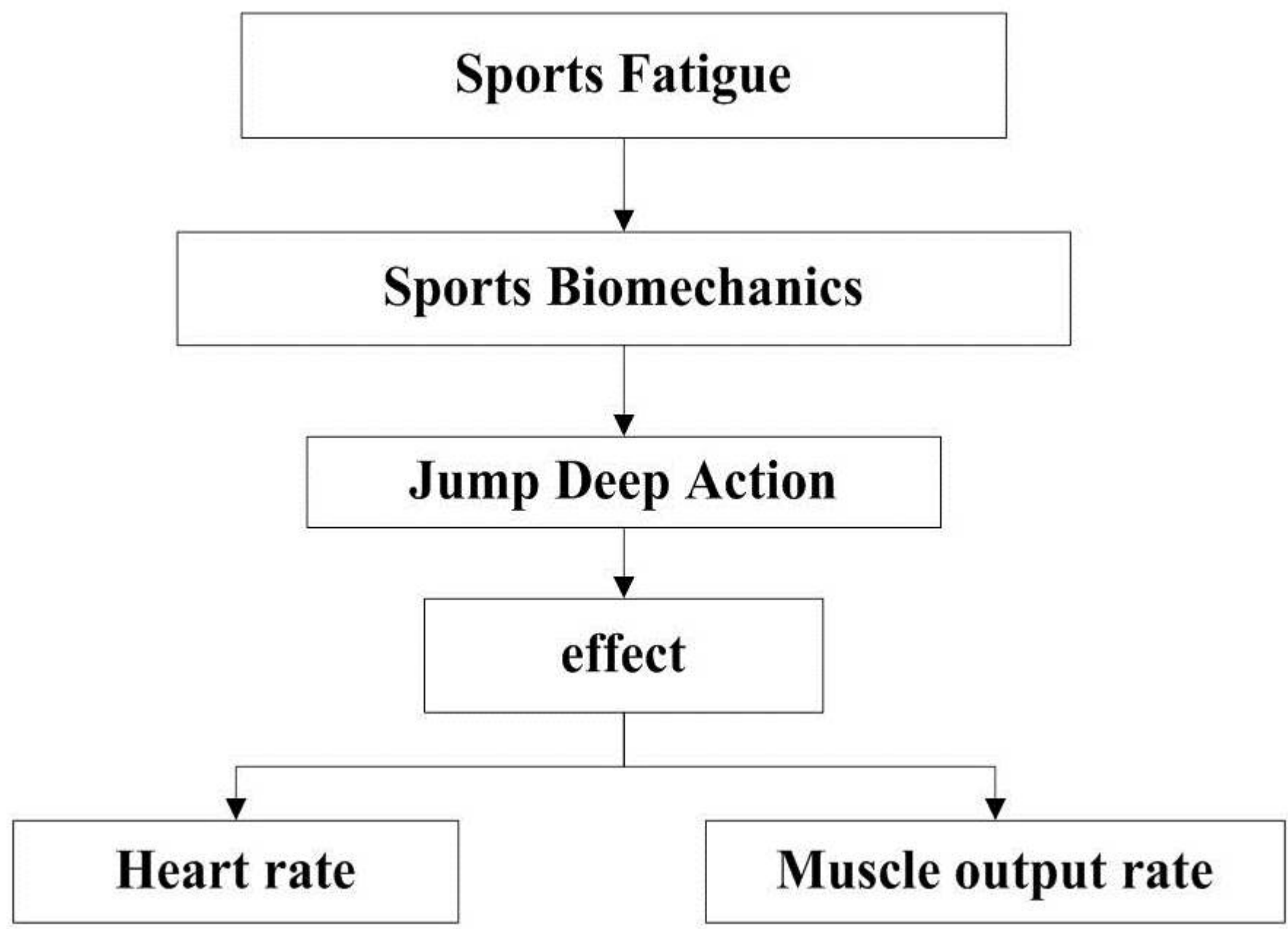

Figure 2. Structure diagram of the impact on the deep jump movement before and after the exercise fatigue based on sports biomechanics.

Based on the above model, it can be seen that the impact of the deep leap movement before and after the exercise fatigue based on sports biomechanics mainly is on the physical parameters, and the impact of this study is mainly in the heart rate and muscle output efficiency in two areas. Excessive fatigue can speed up the heart rate and reduce muscle output efficiency, increase the pressure on the heart.

\section{Result Analysis and Discussion}

In this comparative study, the two groups of athletes were selected for the deep leap action training of the twenty groups, each group had 10 persons. The group A included the athletes who finished the ten deep leap actions, and the group B included the athletes who did not finish the training. The following Figure 3 is a comparison of the two groups of heart rate and muscle output power after the twenty groups of deep leap action. 
As can be seen from Figure 3, the athletes who had been trained in the ten groups after the deep leap exercise were more likely to have sports fatigue than those who had not been trained. After the superposition of the early sports fatigue and the deep leap training, the heart rate increased from the perspective of the sports biomechanics, at the same time, the output power of the muscle was reduced, and the muscle output power was reduced by about $10 \%$. So previous training had an effect on the two indices of heart rate and muscle output rate.

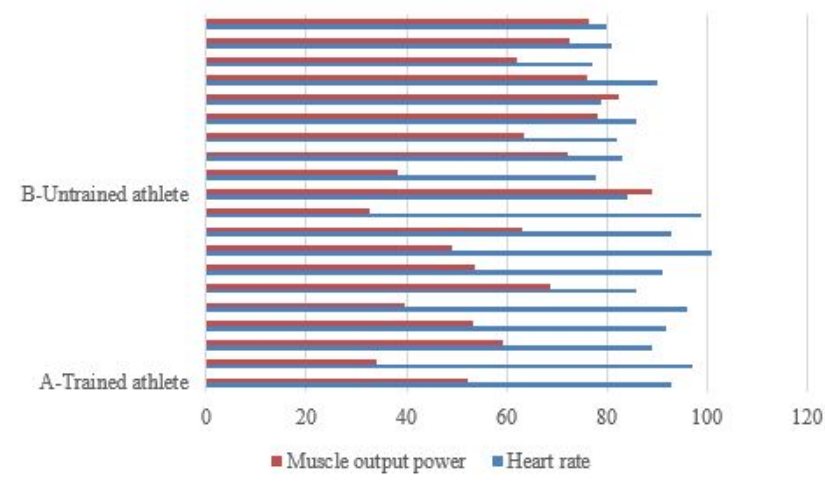

Figure 3. Comparison of heart rate and muscle output power between trained and untrained athletes.

\section{Conclusions}

With the emphasis on sports health and the rapid development of sports biomechanics, sports researchers have devoted themselves to the research of sports health. Based on this, this paper studied the impact on the deep leap movement before and after the exercise fatigue based on sports biomechanics. After a brief introduction of the current situation of the development of sports biomechanics and sports fatigue, this paper introduced the influence of the current sports, especially the deep leap. On the basis of the above analysis, the impact on the deep leap movement before and after the exercise fatigue based on sports biomechanics was analyzed. Finally, the deep leap exercise was taken as an example, the comparative analysis of the heart rate and the muscle output rate of the previous and post fatigue athletes and the non-fatigued athletes was carried out. The results show that the early exercise fatigue can cause an increase in heart rate and a decrease in muscle output. However, this study is still in the experimental stage, and the test samples are less accidental, therefore, this system should be adjusted accordingly.

\section{Acknowledgement}

The study was supported by the Sport bureau of shaanxi procince 2013057.

\section{References}

1. Yoshikawa TS, Mo R. The effects of muscle fatigue on bone strain. J Exp Biol 1994; 188: 217-233.

2. Komi PV, Bosco C. Utilization of stored elastic energy in leg extensor muscles by men and women. Med and Sci in Spor 1978; 10: 261-265.

\section{*Correspondence to}

Lixun $\mathrm{Hu}$

Department of Physical Education,

Shaanxi University of Chinese Medicine, 712046,

PR China 